BULL. AUSTRAL. MATH. SOC.

\title{
On irreducible spaces
}

\section{James R. Boone}

A constructive proof of a theorem of Worrell and Wicke, which states that every open covering of a $\theta$-refinable space has a $\sigma$-distributively point-finite open refinement that covers the space minimally, is presented. Spaces for which every open covering has an open refinement which covers the space minimally are characterized by the use of discrete closed collections and some open questions relating to spaces of this type are included.

\section{Introduction}

A topological space will be called irreducible provided every open covering of the space has an open refinement that covers the space minimally. (That is, no proper subcollection covers the space.) Arens and Dugundji [1] effectively used irreducibility in showing that the metacompact spaces are irreducible and that a space is compact if and only if it is countably compact and metacompact. Two recent contributions to the study of irreducible spaces have been published by Christian, [6] and [7]. Christian calls spaces of this type "minimal cover refinable" and provides a proof that the subparacompact spaces are irreducible.

Worrell and Wicke state, without proof, [8, Theorem (ii)] that the $\theta-r e f i n a b l e ~ s p a c e s$ are irreducible and the minimal open refinement is $\sigma$-distributively point-finite. By the characterization of metacompactness in $\theta$-refinable spaces [3, Theorem 3.1], Theorems (i) and (iii) of [8] follow from other theorems, because both countably compact spaces and collectionwise normal spaces are pointwise collectionwise normal. Due to the structural difference between the metacompact spaces and the

Received 7 November 1974 . 
$\theta-$ refinable spaces, the existential approach employed by Arens and Dugundj does not lend itself to the proof of Worrell and Wicke's Theorem (ii).

It is the primary purpose of this paper to provide a proof, by construction, of Worrell and Wicke's Theorem (ii). Accordingly, many of the results of Christian can be restated in the setting of $\theta$-refinable and weakly $\theta$-refinable spaces [2]. A characterization of irreducible spaces in terms of discrete collections of closed sets is presented. Some open questions relating to irreducibility are posed at the end of this paper. For a collection of sets $M, M^{*}$ will denote the union of the sets in $M$.

\section{Theorems}

THEOREM 2.1 [Worrell and Wicke]. Every -r-refinable space is irreducible.

Proof. Let $G=\left\{G_{\alpha}: \alpha \in A\right\}$ be an open covering of a $\theta$-refinable space $x$. Let $u=\left\{u_{i}: i \in N\right\}$, where $u_{i}=\left\{v_{\alpha}^{i}: \alpha \in A_{i} \subset A, U_{\alpha}^{i} \neq \emptyset\right\}$, be a $\sigma$-precise $\theta$-refinement of $G$. Suppose $A$ is well-ordered. For $k, n \in N$, let

$$
K_{k n}=\left\{p \in X: p \text { is an element of } n \text { or less sets in } u_{k}\right\} \text {. }
$$

The ordering, $<_{0}$, of the double subscripts is defined as follows:

$k n<j l$, if $k_{k n}$ precedes $k_{j l}$ in the sequence,

$k_{11}, K_{21}, K_{12}, K_{31}, K_{22}, K_{13}, k_{41}, \ldots, K_{1 m}, K_{m+1,1}, \ldots$

$$
K_{k n}, K_{k-1, n+1}, \cdots \cdot
$$

(This is the sequence used in the proof of Theorem 3.1 of [3].) For each $\gamma \subset A_{k}$ such that $\operatorname{card}(\gamma)=n$, Iet $H_{\gamma}^{k n}=K_{k n} \cap\left\{n\left\{U_{\alpha}^{k}: \alpha \in \gamma\right\}\right)$. Consider only $H_{\gamma}^{k n}$ which are not empty. For each $\alpha \in A_{k}$, let

$$
T_{\alpha}^{k n}=U\left\{{ }_{H}^{k n}: \alpha \text { is the least element in } \gamma\right\} \text {. }
$$

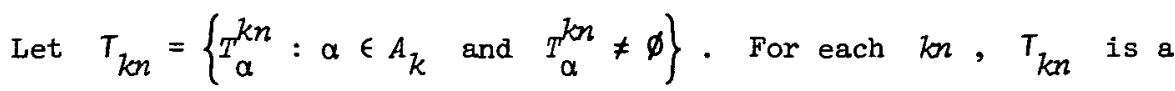


collection of subsets of $X-\underset{j<n}{U} K_{k j}$ which are closed and discrete in $X-\bigcup_{j<n} K_{k j}$. The minimal cover is constructed by induction on the ordered set of double subscripts. For each $\alpha \in A_{1}$, let

$$
V_{\alpha}^{11}=G_{\alpha}-U\left\{T_{\beta}^{11}: \beta \neq \alpha, \beta \in A_{1}\right\}
$$

Let $v_{11}=\left\{v_{\alpha}^{11}: \alpha \in A_{1}, v_{\alpha}^{11} \neq \emptyset\right\}$. For each $\alpha \in A_{2}$, let

$$
v_{\alpha}^{21}=G_{\alpha}-\left(T_{11}^{*} \cup \cup\left\{T_{\beta}^{21}: \beta \neq \alpha, \beta \in A_{2}\right\}\right\} \text {. }
$$

Let

$$
v_{21}=\left\{v_{\alpha}^{21}: \alpha \in A_{2}, v_{\alpha}^{2 l} \cap\left(T_{\alpha}^{2 l}-v_{11}^{*}\right) \neq \emptyset\right\} .
$$

In general, for any $k n$, let

$$
v_{\alpha}^{k n}=G_{\alpha}-\left(\left(U\left\{T_{i j}^{*}: i j<_{0} k n\right\}\right) \cup \cup\left\{T_{\beta}^{k n}: \beta \neq \alpha, \beta \in A_{k}\right\}\right),
$$

for each $\alpha \in A_{k}$. Let

$$
v_{k n}=\left\{v_{\alpha}^{k n}: \alpha \in A_{k}, v_{\alpha}^{k n} n\left\{T_{\alpha}^{k n}-U\left\{V_{i j}^{*}: i j<_{0} k n\right\}\right) \neq \emptyset\right\} .
$$

Then $V=U V_{i j}$ is a minimal open covering of $X$ which refines $G$. Since $V$ is clearly an open covering of $X$ that refines $G$, the minimality of $V$ will be verified. Let $v_{\alpha}^{k n}$ be any set in $V$. Since

$$
v_{\alpha}^{k n} \cap\left(T^{k n}-U\left\{v_{i j}^{*}: i j<0 k n\right\}\right) \neq \emptyset
$$

and

$$
T_{\alpha}^{k n}-\left\{U\left\{T_{i j}^{*}: i j<0 k n\right\} \cup U\left\{T_{\beta}^{k n}: \beta \neq \alpha, \beta \in A_{k}\right\}\right) \subset v_{\alpha}^{k n},
$$

the non-empty set $T_{\alpha}^{k n}-U\left\{V_{i j}^{*}: i j<<_{0} k n\right\}$ is covered by $V_{\alpha}^{k n}$. Since $\left\{T_{\alpha}^{k n}-U\left\{V_{i j}^{*}: i j<_{0} k n\right\}\right) \cap v_{B}^{t r}=\varnothing$ for each $t r<_{0} k n$ and for each $\beta \in A_{t}, V_{\beta}^{k n} \cap T_{\alpha}^{k n}=\varnothing$ if $\beta \neq \alpha$ and if $k n<<_{0} t r, v_{B}^{t r} \cap T_{\alpha}^{k n}=\varnothing$ for 
each $\beta \in A_{t}, V_{\alpha}^{k n}$ is the only set in $V$ that covers the non-empty set $T_{\alpha}^{k n}-U\left\{V_{i j}^{*}: i j<_{0} k n\right\}$. Thus $V$ is minimal and this completes the proof.

Note that the refinement $V$ in the preceding,proof is a $\sigma$-distributively point-finite refinement of $G$, as Worrell and Wicke indicate.

The preceding theorem is an improvement of Theorem 1 of [6]. The main results of Christian's paper [6] are: an irreducible space is Lindelöf if and only if it is $\aleph_{1}$-compact, and hereditarily separable irreducible spaces are Lindelöf. The hereditary properties of irreducible spaces are not as simple as one might expect, as the discussion in Section 3 of [7] indicates. In particular, irreducibility is not weakly hereditary. However, since weakly $\theta$-refinable spaces with closed sets $G_{\delta}$ are hereditarily subparacompact, the following additional comment can be made relative to Theorem 3.5 of [7].

COROLLARY 2.2. Every weakly $\theta$-refinable space with closed sets $G_{\delta}$ is hereditarily irreducible.

The following characterization indicates the intrinsic relationship between irreducibility and the cardinality of open subcoverings with regard to the cardinality of discrete collections of closed sets.

THEOREM 2.3. A space $X$ is irreducible if and only if for each open covering $\left\{V_{\alpha}: \alpha \in A\right\}$ of $X$ there exists a discrete collection of nonempty closed sets $\left\{T_{\beta}: B \in B\right\}$ such that $B \subset A, T_{\beta} \subset V_{\beta}$ for each $\beta \in B$ and $\left\{V_{\beta}: \beta \in V\right\}$ covers $X$.

Proof. Let $V=\left\{V_{\alpha}: \alpha \in A\right\}$ be an open covering of an irreducible space $X$. Let $\left\{U_{\alpha}: \alpha \in B \subset A, U_{\alpha} \neq \varnothing\right\}$ be a precise minimal open refinement of $V$ that covers $X$. For each $\beta \in B$, let $T_{\beta}=X-U\left\{U_{\alpha}: \alpha \neq \beta\right\}$. Then $T=\left\{T_{\beta}: \beta \in B\right\}$ is a discrete collection of non-empty closed sets such that $T_{\beta} \subset V_{\beta}$ for each $\beta \in B$, and $\left\{V_{\beta}: B \in V\right\}$ covers $X$. To prove the converse, let $V=\left\{V_{\alpha}: \alpha \in A\right\}$ be 
an open covering of a space $X$. Let $T=\left\{T_{\beta}: B \in B\right\}$ be a discrete collection of non-empty closed sets such that $B \subset A, T_{\beta} \subset V_{\beta}$ for each $\beta \in B$, and $\left\{V_{\beta}: \beta \in B\right\}$ covers $X$. For each $\beta \in B$, let $U_{\beta}=V_{\beta}-U\left\{T_{\alpha}: \alpha \neq \beta\right\}$. Then $\left\{U_{\beta}: \beta \in B\right\}$ is an open refinement of $V$ that covers $X$ minimally. This completes the proof.

The weakly $\theta$-refinable, but not $\theta$-refinable modifications of Bing's Example $G$, presented by Burke [5], remain irreducible. Variations of the constructive proof of Theorem 2.1 have not yielded an answer to the question:

Is every weakly $\theta$-refinable space irreducible?

An affirmative answer to this question would answer two more restricted questions:

Is an $\aleph_{1}$-compact weakly $\theta$-refinable space Lindelöf?

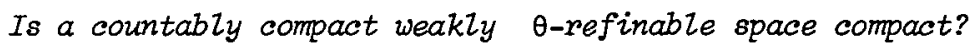

Also, the mapping properties of $\theta$-refinable and weakly $\theta$-refinable spaces remain as open questions. It is known that Arhangel'skir's class MOBI contains a space which is not countably $\theta$-refinable [4], but whether every space in MOBI is weakly $\theta$-refinable remains unanswered. Thus the additional question arises:

What are the mapping properties of irreducible spaces?

In particular, is every space in Arhangel'skii's class MOBI irreducible?

\section{References}

[1] Richard Arens and J. Dugundji, "Remark on the concept of compactness", Portugal. Math. 9 (1950), 141-143.

[2] H.R. Bennett and D.J. Lutzer, "A note on weak $\theta$-refinability", General Topology and Appl. 2 (1972), 49-54.

[3] James R. Boone, "A characterization of metacompactness in the class of O-refinable spaces", General Topology and Appl. 3 (1973), 253-264. 
[4] James R. Boone, "An example relating to Arhangel'skii's class MOBI", Proc. Amer. Math. Soc. 41 (1973), 615-618.

[5] Dennis K. Burke, "A note on R.H. Bing's Example G", Topology Conference, 47-52 (Proc. Conf. Virginia Polytechnic Institute and State University, 1973. Lecture Notes in Mathematics, 375. Springer-Verlag, Berlin, Heidelberg, New York, 1974).

[6] Upton J. Christian, "A note on the relation between Lindelöf and $\aleph_{1}$-compact spaces", Comment. Math. Prace Mat. 16 (1972), 215-217.

[7] U.J. Christian, "Concerning certain minimal cover refinable spaces", Fund. Math. 76 (1972), 213-222.

[8] J.M. Worrell, Jr. and H.H. Wicke, "Characterizations of developable topological spaces", Canad. J. Math. 17 (1965), 820-830.

Department of Mathematics,

Texas A\&M University,

College Station,

Texas, USA. 